The $8^{\text {th }}$ International Conference of the Slovenian Society for Non-Destructive Testing

"Application of Contemporary Non-Destructive Testing in Engineering"

September 1-3, 2005, Portorož, Slovenia, pp. 293-298

\title{
APPLICATION OF PSYCHOACOUSTIC FILTERING FOR MACHINE FAULT DETECTION
}

\author{
P. Potočnik, E. Govekar, I. Grabec \\ Faculty of Mechanical Engineering, Laboratory of Synergetics, \\ Aškerčeva 6, 1000 Ljubljana, Slovenia, \\ E-mails:primoz.potocnik@fs.uni-lj.si, edvard.govekar@fs.uni-lj.si, \\ igor.grabec@fs.uni-lj.si
}

\begin{abstract}
A method for nondestructive machine fault detection, based on evaluation of acoustic machine signatures, is presented. Various mechanical defects of rotary machines can be reflected in altered acoustic signatures. Such phenomena can be often perceived by skilled human operators who can also explain the type of defect merely based on slightly changed acoustic signature. The proposed method is based on psychoacoustic modeling of human auditory perception. For the purpose of machine fault detection, the gammatone filterbank is applied in preprocessing of acoustic signals. Filtered signals are then rectified, and features are calculated as mean values of rectified signals. A set of features represents an extracted machine signature. In order to evaluate the current machine state, extracted features are compared to the database of normal machine states. If values of the extracted features exceed statistically determined upper and lower margins, a possible machine fault is indicated. The proposed approach is illustrated by a case study, where the quality of commercially produced compressors is predicted. Compressor states are estimated based on acoustic emission during operation, and features are extracted by psychoacoustic filtering as described above. Results show that major faults, that occur in a production, can be reliably detected.
\end{abstract}

Keywords: Machine fault detection, Acoustic signals, Psychoacoustic filtering

\section{Introduction}

Modern industrial production based on machining processes requires reliable condition monitoring procedures. By a proper monitoring of machines, a harmful machine breakdown and consequently a loss of production can be prevented. Various sensory signals, such as acoustic emission, temperature, vibration and forces, can be monitored [1] in order to estimate the machine state. Sound analysis was already successfully applied by several authors for the fault detection of rotary machines $[2,3,4]$.

Trained human operators can often estimate machine faults based on simple aural inspection. Our ability of aural analysis is modeled by psychoacoustic models which combine several signal processing stages for simulating aspects of transformation in the auditory periphery [5]. A preprocessing stage combines an array of independent bandpass filters with signal rectification and low-pass filtration. This stage roughly simulates the transformation of mechanical 
oscillations of the basilar membrane into receptor potentials in the inner hair cells. Further stages combine nonlinear adaptation for compression and high sensitivity for fast temporal variations. Finally, signals are analyzed by a decision device.

The objective of this paper is to present an automated aural fault detection system, based on simplified models of human auditory models. We propose the approach for automatic aural detection of faults in machines that emit continuous sounds, and defects occur only as slight alterations of a base sound. Such an example is demonstrated in the case study of fault detection in compressors where defected compressors are recognized by stationary emission of altered buzz pattern. The solution approach is proposed in the next section. Then extraction and evaluation of features are described, and finally, a case study is presented.

\section{Solution approach}

Solution approach to the aural detection of faults in machines that emit continuous sounds, is proposed. In the case of a continuous sound emission, sound signals can be averaged over short time intervals and treated as stationary. For machine fault detection applications, only the preprocessing stage of the auditory model is retained. The method consists of feature extraction stage, and feature evaluation stage. The method is presented in Fig. 1. Feature extraction stage includes auditory band-pass filters (BP), rectifiers (R), estimators of mean values (M) and logarithm $(\log )$ calculation. Feature evaluation stage combines comparison of extracted features with the database, detection of faulty conditions, and adaptation of the database. Details are described in the next sections.

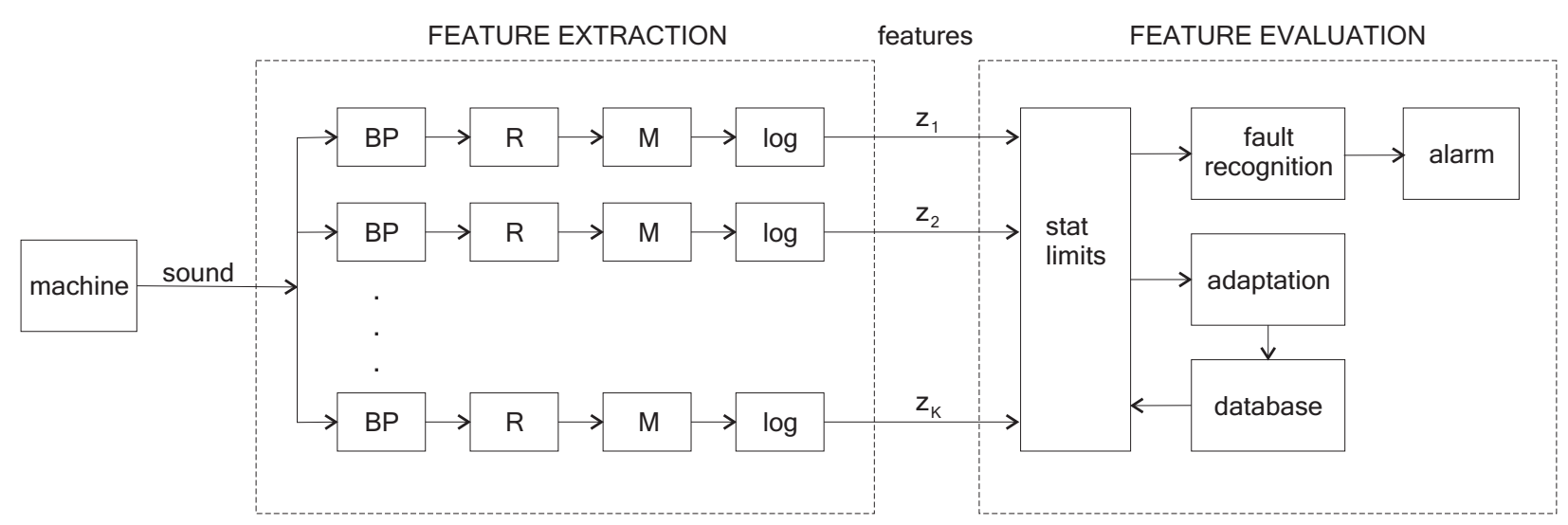

Fig. 1: System for automatic aural fault detection.

\section{Extraction of features}

The purpose of feature extraction stage is to generate a vector of features of a reduced dimension compared to the original signals. Input part of feature extraction stage consists of auditory gammatone filter bank, as proposed by [6]. Gammatone filterbank roughly simulates the transformation of mechanical oscillations of the basilar membrane into receptor potentials in the inner hair cells. The filter bank is designed as a set of parallel band-pass filters, each tuned to a different frequency.

In our analysis, an efficient filter bank implementation [7] was used. Filterbank is defined by the following parameters: sampling frequency $f_{s}$, starting frequency $f_{0}$, and number of filters $N_{f}$. An example of gammatone filter bank $\left(f_{s}=20 \mathrm{kHz}, f_{0}=300 \mathrm{~Hz}, N_{f}=10\right)$ is shown in Fig. 2. 


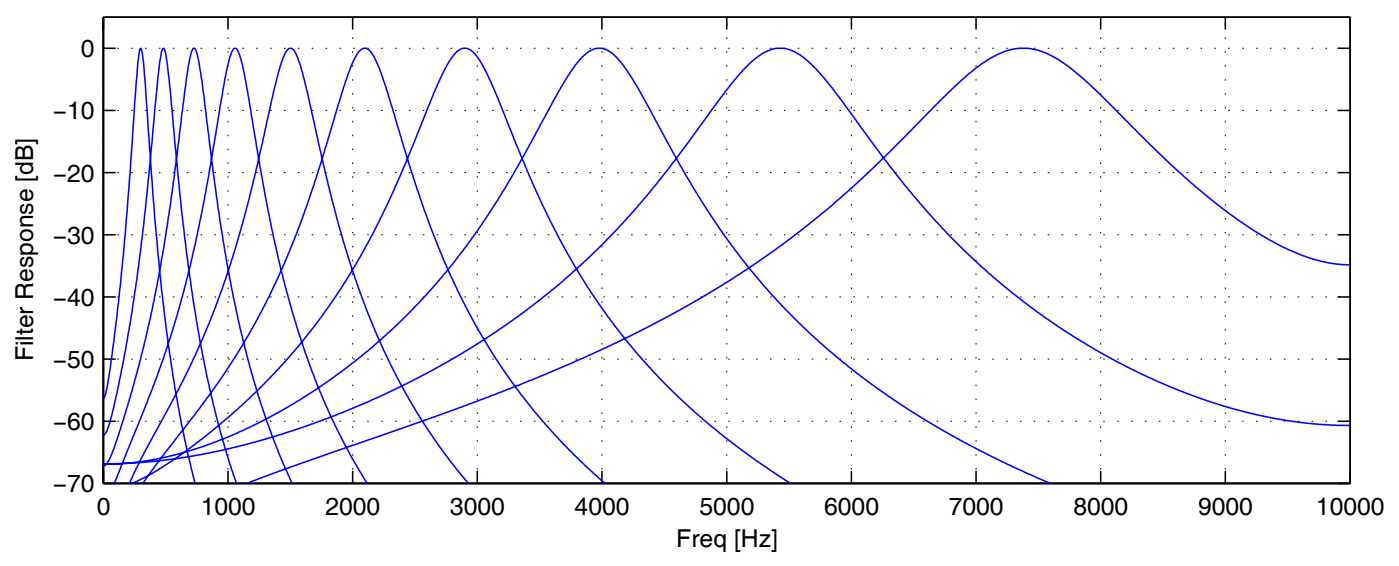

Fig. 2: Gammatone filter bank $\left(f_{s}=20 \mathrm{kHz}, f_{0}=300 \mathrm{~Hz}, N_{f}=10\right)$.

After band-pass filtering, the filtered signals are rectified and then their mean value is calculated. With this step, the average contribution of each band to the overall sound power, is calculated. The final feature extraction step includes logarithmic operator on each band. The purpose of this step is to normalize the distribution of features, therefore, this step is only optional. The result of feature extraction stage is a set of features $\left(z_{1}, z_{2}, \ldots, z_{K}\right)$ which are evaluated in the next stage of processing.

\section{Evaluation of features}

In order to detect machine failures, extracted features must be properly evaluated. We propose statistical comparison of extracted features with a database of recorded features. The 1-class classifier case will be considered where only features extracted from good samples are available. Each feature from the feature set $\left(z_{1}, z_{2}, \ldots, z_{K}\right)$ is evaluated separately by statistical comparison with the corresponding feature database.

If the distribution of features is normal, symmetrical $\pm 3 \sigma$ margins can be applied, with $\sigma$ indicating the standard deviation. In this case, features that exceed $\pm 3 \sigma$ margins indicate the possible machine defects. There are two reasons against the application of standard statistical $6 \sigma$ range evaluation:

- Features extracted in many practical applications are not normally distributed, therefore asymmetrical evaluations are encouraged.

- Often, some features are not informative and mainly represent noise. Such features should be eliminated from the fault recognition process and this can be accomplished by substituting the second moment in the calculation of the standard deviation by a higher moment. This results in broadening the margins for very noisy features.

We propose the fault detection margins which are based on separate asymmetrical calculations of high $\left(t_{+}\right)$and low $\left(t_{-}\right)$margin, and are calculated by higher moments of feature median. The margins are defined for each feature separately by the following equations:

$$
\begin{aligned}
& t_{+}=m+s \sqrt[p]{\overline{\left(Z_{+}-m\right)^{p}}} \\
& t_{-}=m-s \sqrt[p]{\overline{\left(m-z_{-}\right)^{p}}}
\end{aligned}
$$

Symbols denote: median value $m$, features above median $z_{+}$, and features below median $z_{-}$. The calculation of margins is controlled by parameters $s$ and $p$ which denote the stretching factor and 
the moment of the feature median. We propose default values $s=3$ and $p=4$. For a normal distribution, values $s=3$ and $p=2$ correspond to $\pm 3 \sigma$ margins.

Examples of setting the fault detection margins for a database of extracted features are shown below. Fig. 3 shows the standard statistical $\pm 3 \sigma$ margins, and Fig. 4 shows the asymmetrical margins obtained by Eq. 1-2. It is evident that $\pm 3 \sigma$ margins in the first case (Fig. 3) are not appropriate choice because several false alarms are indicated. The selection of appropriate margins is considerably improved in the second case (Fig. 4).

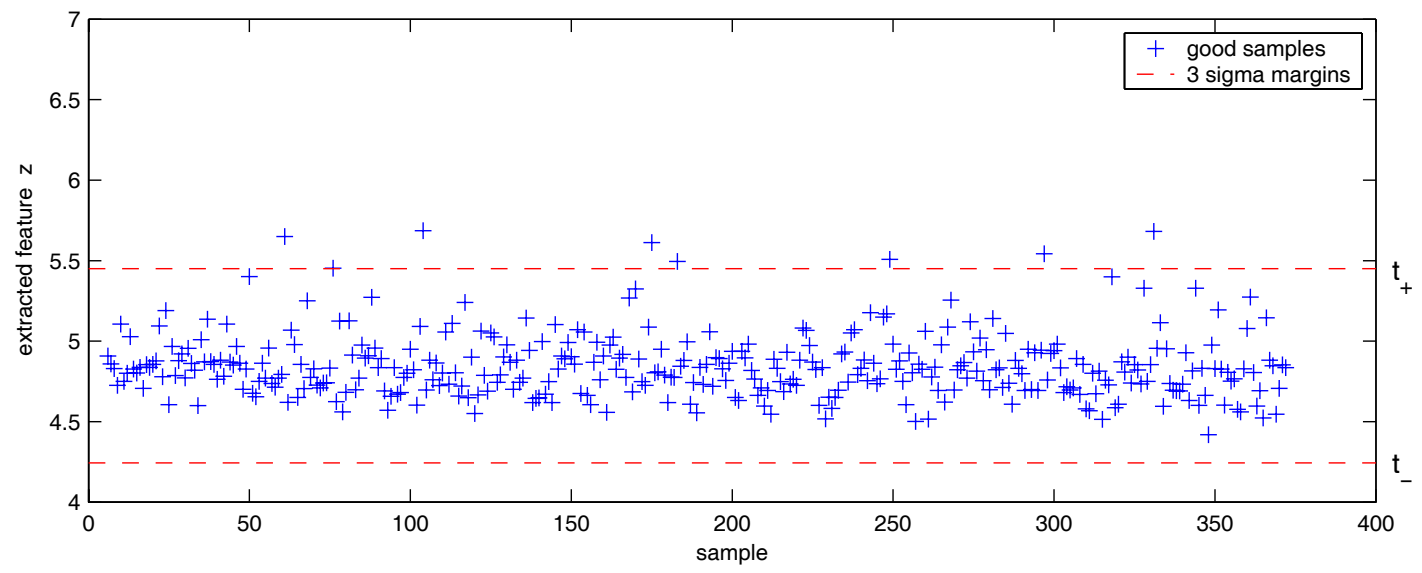

Fig. 3: The population of extracted features with standard statistical $\pm 3 \sigma$ margins.

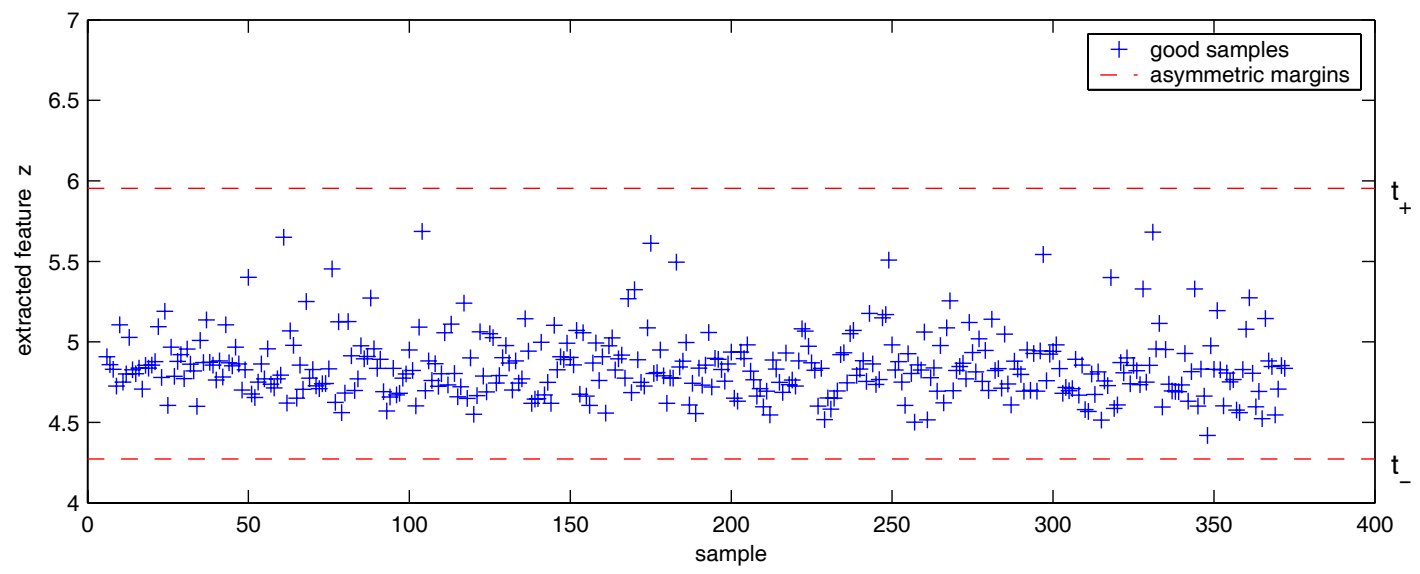

Fig. 4: The population of extracted features with asymmetrical margins $(s=3, p=4)$, obtained by Eq. 1-2.

In this paper, setting of asymmetrical margins is discussed where a whole population from the database of good samples is used for the calculation of margins. However, it should be mentioned that online adaptation of margins based on recent machine states is preferable in a case of frequent fluctuations due to process drift in a changing industrial environment. In such a case, most recent samples from the database have more impact on the calculation of margins.

The last step of the feature evaluation stage is the adaptation of the database by novel samples. If evaluated sample is recognized as a "normal" one, the extracted features of this sample are appended to the database. Thus, the calculation of asymmetrical margins on the next evaluation step will base on the refreshed database. 


\section{Case study: fault detection in compressors}

The proposed method is applied to a case study of fault detection in compressors. Industrial production of compressors in company Danfoss Compressors, d.o.o. (Slovenia) requires an accurate fault detection system at the end of the production line. Finalized compressors can only be inspected by non-destructive methods that is at present accomplished by aural monitoring by skilled operators. The present case study demonstrates the possibilities of automatic aural sound detection, based on the proposed method.

In this example, two types of defects are considered:

- noxious space defect

- lubrication defect

The experiments were performed on the production line in the company Danfoss Compressors, d.o.o. A set of compressors with built-in defects was prepared and inserted into the normal production line. Acoustic signals, recorded at the top of the operating compressor, were recorded on a set of 332 normal compressors, 20 compressors with noxious space defect, and 20 compressors with lubrication defect. Signals were acquired in the time interval [0.3-1.3] seconds after switching the compressor on.

The objective of this task is to automatically recognize a faulty compressor based on its acoustic signature. Acoustic signals of each compressor are analyzed by the proposed solution. Features are extracted by psychoacoustic filtering $\left(f_{s}=20 \mathrm{kHz}, f_{0}=300 \mathrm{~Hz}, N_{f}=10\right)$ and results are shown in Fig. 5 together with asymmetrical fault detection margins $(s=3, p=4)$.
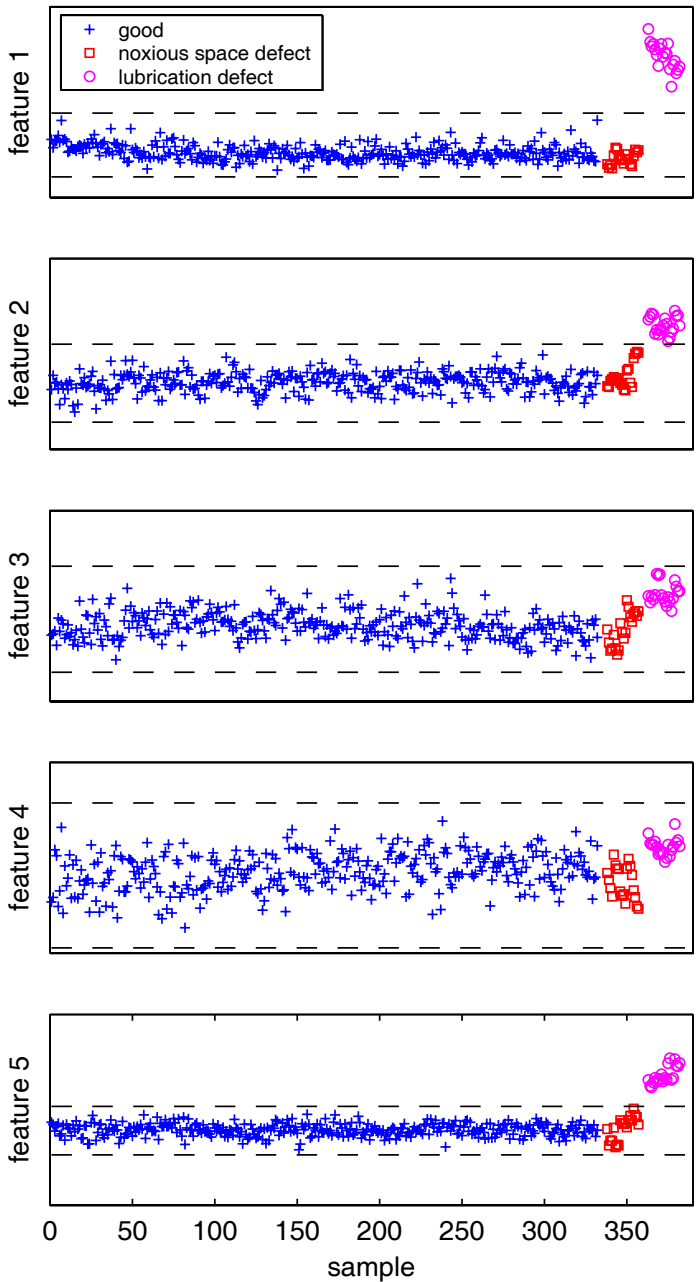
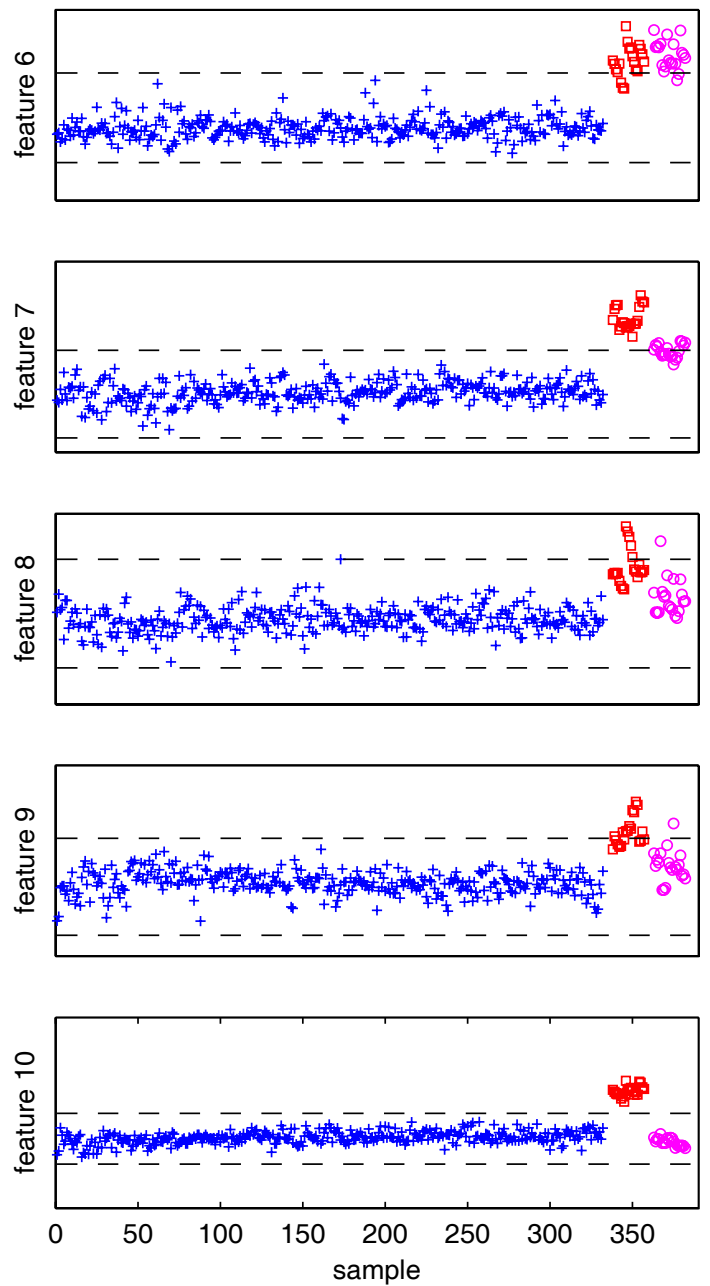
Fig. 5: Extracted features with asymmetrical evaluation margins for the set of normal compressors, compressors with noxious space defect, and compressors with lubrication defect. Fig. 5 shows that a noxious space defect is automatically detected by features 7 and 10 . Lubrication defect is best detected by features 1 and 5. All defected compressors are successfully recognized without triggering false alarms. Extracted features 3 and 4 are noisy and do not contribute toward the fault detection. For noisy features, it is important not to trigger false alarms and this is accomplished by applying the proposed feature evaluation procedure. It is evident from Fig. 5 that the activity of various features also indicates the type of defect and not only normal or defected condition.

\section{Conclusions}

The method for automatic aural detection of machine faults is presented. Solution approach combines feature extraction stage and feature evaluation stage. Feature extraction stage is implemented by psychoacoustic filtering of the acquired sound signals and simple signal conditioning, and results in features which represent acoustic power in each band. Asymmetrical margins are proposed for the evaluation of extracted features with the objective to detect machine defects and to prevent false alarms. The presented case study demonstrates the efficiency of the proposed method. Various compressor defects that are difficult to recognize by aural inspection of skilled operators, are successfully detected. The method is suitable for a broad class of machining processes that emit continuous sound patterns.

\section{7. $\quad$ References}

[1] Dimla Snr. D.E.: Sensor signals for tool-wear monitoring in metal cutting operations - a review of methods, International Journal of Machine Tool \& Manufacture, Vol. 40, 2000, 1073-1098.

[2] Benko U., Petrovčič J., Juričič D., Tavčar J., Rejec J., Stefanovska A.: Fault diagnosis of a vacuum cleaner motor by means of sound analysis, Journal of Sound and Vibration, Vol. 276, 2004, 781-806.

[3] Lin J.: Feature extraction of machine sound using wavelet and its application in fault diagnosis, NDT\&E International, Vol. 34, 2001, 25-30.

[4] Tsao T.P., Wu R.C.: The application of artificial neural network on sound signal recognition for induction motor. In: Proc. Natl. Sci. Counc. ROC(A), Vol. 23, 1999, 7584.

[5] Dau T., Püschel D.: A quantitative model of the "effective" signal processing in the auditory system. I. Model structure. The Journal of the Acoustical Society of America, Vol. 99, 1996, 3615-3622.

[6] Patterson R.D., Robinson K., Holdsworth J., McKeown D., Zhang C., Allerhand M.H.: Complex sounds and auditory images, in: Auditory Physiology and Perception (Cazals Y., Demany L., Horner K., eds.), Pergamon, Oxford, 1992, 429-446.

[7] Slanley M.: An Efficient Implementation of the Patterson-Holdsworth Auditory Filter Bank. Apple Computer Technical Report \#35, Perception Group-Advanced Technology Group, Apple Computer, Inc., 1993. 